\title{
A Short Commentary on Non-invasive Brain Stimulation in Chronic Disorder of Consciousness Differential Diagnosis
}

\section{Antonino Naro* and Rocco Salvatore Calabrò}

IRCCS Centro Neurolesi “Bonino-Pulejo”, Messina, Italy

*Corresponding author: Antonino Naro, IRCCS Centro Neurolesi "Bonino-Pulejo" S.S. 113, Contrada Casazza, 98124, Messina, Italy, Tel: +3909060128840; E-mail: g.naro11@alice.it

Received date: April 12, 2016; Accepted date: May 09, 2016; Published date: May 16, 2016

Copyright: (C) 2016 Naro A, et al. This is an open-access article distributed under the terms of the Creative Commons Attribution License, which permits unrestricted use, distribution, and reproduction in any medium, provided the original author and source are credited.

\begin{abstract}
The diagnosis and awareness assessments in chronic disorders of consciousness (DOC) are biased, owing to their reliance on clinical behavioral measures. Indeed, objective tools should be employed with the goal of more precise awareness detection in these patients. To this end, brain stimulation techniques have been developed to demonstrate residual cortical-thalamocortical connectivity patterns sustaining fragment of awareness. This short commentary aims at illustrating the Non-Invasive Brain Stimulation (NIBS) techniques applied to DOC patients. NIBS are promising in helping clinicians to differentiate among DOC patients and to identify residual fragments of awareness within Vegetative State individuals, namely Functional Locked-In Syndrome.
\end{abstract}

Keywords: Chronic disorders of consciousness; Functional locked-in syndrome; Minimally conscious state; Non-invasive brain stimulation; Vegetative state

\section{Introduction}

Chronic disorders of consciousness (DOC), including Minimally Conscious State (MCS) and Vegetative State (VS) [1], are characterized by a deterioration of awareness (i.e., an impairment of purposeful behavioral responsiveness) that arises from severe brain injuries producing multifocal neuronal cell death and inter-area disconnection $[2,3]$. In summary, awareness impairment depends on a disruption of the basic connectivity patterns within the entire corticalthalamocortical system (according to the Schiff's mesocircuit model), which are associated with different levels of consciousness [4-6]. The metabolic activity and the functional connectivity of specific parts of such a system (e.g., the precuneus and thalamus) have been shown to correlate with the levels of expressed behaviors and recovery in DOC patients [4,7]. Nonetheless, the postulated correlation between thalamocortical connectivity and behavioral responsiveness is not always straightforward. In fact, some DOC patients, namely the individuals suffering from Functional Locked-In Syndrome, may have a covert awareness but be unable to show purposeful behaviors because of severe motor output impairment, despite the thalamocortical system is functionally preserved [8].

Non-Invasive Brain Stimulation (NIBS) may represent a possible approach to differentiate the patients with covert awareness from the VS individuals. NIBS comprise a wide repertoire of instruments, including transcranial magnetic -TMS- and electric stimulation, which can shape synaptic plasticity and neural network connectivity [9]. TMS induces electrical currents within the brain through the Faraday's principle of electromagnetic induction. TMS is used to evaluate the integrity of the corticospinal tract, spinal cord, and peripheral nerves. In addition, repetitively employed TMS can induce plastic changes in the brain, modulate the activity of distant, functionally connected brain regions, and organize neuronal networks [9]. Among transcranial electric stimulations, the transcranial direct current stimulation (tDCS) is far back used as a NIBS technique. tDCS consists of a flow of weak currents (1-2 mA) from an anode to a cathode, circumscribing a brain area target. The delivered electrical current modifies the resting membrane potential and the activity level of spontaneous excitatory neurons [10].

When dealing with DOC patients, NIBS should be aimed at restoring specific and non-specific neurotransmission patterns within cortical-thalamocortical networks and the Schiff's mesocircuit model, which may release the thalamocortical outflow from central thalamus to the cortical targets, thus improving the level of awareness $[5,11]$. Both TMS and tDCS have been thought to interfere with long longterm potentiation and long-term depression mechanisms of synaptic plasticity [12], thus shaping the neuronal firing, synaptic strength, neurotransmitter release, and neural network connections [9]. Therefore, NIBS on specific brain targets may allow highlighting the crucial role of neuronal circuit mechanisms underpinning conscious state control, and to demonstrate indirectly the functional preservation of broad thalamocortical networks potentially sustaining covert awareness [13]. Indeed, it has been shown in DOC samples that TMS and $\mathrm{tDCS}$ can unmask residual covert connectivity patterns in parallel to a behavioral responsiveness improvement [14,15]. Moreover, audiomotor and visuomotor stimulation could be promising tools to potentiate the functional connectivity within large-scale sensorymotor networks [16,17]. These new approaches exploring covert behaviors and functional connectivity may yield further insight into DOC pathophysiology and improve the differential diagnosis.

In conclusion, NIBS may play a major role in inducing neuroplasticity within residual brain networks potentially sustaining awareness, thus allowing differentiating DOC patients. Moreover, there is growing evidence that NIBS has a great potential for improving the awareness level [11], with a potentially strong impact on the current rehabilitation protocols of DOC patients. 
Citation: Naro A, Calabrò RS (2016) A Short Commentary on Non-invasive Brain Stimulation in Chronic Disorder of Consciousness Differential Diagnosis. J Neurol Neurophysiol 7: 372. doi:10.4172/2155-9562.1000372

Page 2 of 2

\section{References}

1. Blume C, Del Giudice R, Wislowska M, Lechinger J, Schabus M (2015) Across the consciousness continuum-from unresponsive wakefulness to sleep. Front Hum Neurosci 9: 105.

2. Laureys S, Schiff ND (2012) Coma and consciousness: paradigms (re)framed by neuroimaging. Neuroimage 61: 478-491.

3. Giacino JT, Fins JJ, Laureys S, Schiff ND (2014) Disorders of consciousness after acquired brain injury: the state of the science. Nat Rev Neurol 10: 99-114.

4. Fernández-Espejo D, Soddu A, Cruse D, Palacios EM, Junque C, et al. (2012) A role for the default mode network in the bases of disorders of consciousness. Ann Neurol 72: 335-343.

5. Schiff ND (2010) Recovery of consciousness after brain injury: a mesocircuit hypothesis. Trends Neurosci 33: 1-9.

6. Haider B, McCormick DA (2009) Rapid neocortical dynamics: cellular and network mechanisms. Neuron 62: 171-189.

7. Vanhaudenhuyse A, Noirhomme Q, Tshibanda L, Bruno M, Boveroux P, et al (2010) Default network connectivity reflects the level of consciousness in non-communicative brain-damaged patients. Brain 133 161-171.

8. Formisano R, D'Ippolito M, Catani S (2013) Functional locked-in syndrome as recovery phase of vegetative state. Brain Inj 27: 1332.

9. Demirtas-Tatlidede A, Vahabzadeh-Hagh AM, Bernabeu M, Tormos JM, Pascual-Leone A (2012) Noninvasive brain stimulation in traumatic brain injury. J Head Trauma Rehabil 27: 274-292.

10. Woods AJ, Antal A, Bikson M, Boggio PS, Brunoni AR, et al. (2016) A technical guide to tDCS, and related non-invasive brain stimulation tools. Clin Neurophysiol 127: 1031-1048.
11. Li S, Zaninotto AL, Neville IS, Paiva WS, Nunn D, et al. (2015) Clinical utility of brain stimulation modalities following traumatic brain injury: current evidence. Neuropsychiatr Dis Treat 11: 1573-1586.

12. Liebetanz D, Nitsche MA, Tergau F, Paulus W (2002) Pharmacological approach to the mechanisms of transcranial DC stimulation-induced after-effects of human motor cortex excitability. Brain 125: 2238-2247.

13. Voss HU, UluÄŸ AM, Dyke JP, Watts R, Kobylarz EJ, et al. (2006) Possible axonal regrowth in late recovery from the minimally conscious state. J Clin Invest 116: 2005-2011.

14. Naro A, Calabrò RS, Russo M, Leo A, Pollicino P, et al (2015) Can transcranial direct current stimulation be useful in differentiating unresponsive wakefulness syndrome from minimally conscious state patients? Restor Neurol Neurosci 33: 159-176.

15. Naro A, Russo M, Leo A, Bramanti P, Quartarone A, et al. (2015) A Single Session of Repetitive Transcranial Magnetic Stimulation Over the Dorsolateral Prefrontal Cortex in Patients With Unresponsive Wakefulness Syndrome: Preliminary Results. Neurorehabil Neural Repair 29: 603-613.

16. Naro A, Leo A, Cannavò A, Buda A, Bruno R, et al. (2015) Audiomotor Integration in Minimally Conscious State: Proof of Concept! Neural Plast 2015: 391-349.

17. Naro A, Leo A, Filoni S, Bramanti P, Calabrò RS (2015) Visuomotor integration in unresponsive wakefulness syndrome: A piece of the puzzle towards consciousness detection? Restor Neurol Neurosci 33: 447-460. 